\title{
Desensitization as a tool for beta-lactam antibiotic use in methicillin sensitive Staphylococcus aureus infections
}

\author{
Julie H. Cash ${ }^{1 *}$, Celeste Rudisill Caulder ${ }^{2}$, April Miller Quidley ${ }^{3}$, Mary Carolyn Frith ${ }^{2}$, Alicia Davenport ${ }^{2}$ and P. Brandon Bookstaver ${ }^{2}$ \\ *Correspondence: cashj@shands.ufl.edu \\ ${ }^{1}$ Clinical Pharmacist, Critical Care Shands at the University of Florida, 1600 SW Archer Road Gainesville, FL 32610, USA. \\ 2Department of Clinical Pharmacy and Outcomes Sciences, South Carolina College of Pharmacy-USC Campus, 715 Sumter \\ Street Columbia, SC 29208, USA. \\ ${ }^{3}$ Clinical Pharmacist, Critical Care Vidant Medical Center PO Box 6028 Greenville, NC 27835, USA.
}

\begin{abstract}
Purpose: The purpose of this case report is to describe the successful application of a cefazolin desensitization protocol in a penicillin allergic patient and to discuss the potential advantages for the use of desensitization protocols in commonly encountered Staphylococcal infections.

Summary: A 64 year old Caucasian man presented to the hospital with lower extremity pain and petechiae. Allergies documented in the medical chart at admission include amoxicillin and cefotetan. The patient was started on vancomycin 1 gram every 12 hours $(14 \mathrm{mg} / \mathrm{kg}$ ) empirically which was continued for 14 days. Two out of 2 blood cultures revealed MSSA. The patient's signs and symptoms improved and he was discharged on hospital day 16 after completing 14 days of vancomycin therapy for his MSSA bacteremia. Less than 72 hours after discharge, the patient presented again to the hospital with increasing lower extremity edema and fever. Infectious diseases consult on day 2 of hospitalization recommended continuing vancomycin due to abscess the documented reaction of severe itching with penicillin. On day 5, surgical debridement of the lower extremity abscesses was performed and the infectious diseases team recommended cefazolin desensitization followed by a 2 -week course of cefazolin. The patient tolerated the desensitization protocol and was continued on cefazolin 1 gram every 8 hours for 14 days. The patient improved significantly and repeat blood cultures on day 18 of hospitalization were negative.

Conclusion: For patients that have a true allergy to penicillin, desensitization should be a consideration to facilitate beta-lactam therapy in the management of patients with invasive MSSA infections. When performed appropriately, desensitization can be a safe alternative with minimal risk for serious adverse reactions.
\end{abstract}

Keywords: Desensitization, beta-lactam, cefazolin, MSSA

\section{Introduction}

Self-reporting of antimicrobial allergies among hospitalized patients is quite common, with up to $25 \%$ of patients reporting at least one antimicrobial allergy [1]. While it is important that patients report all suspected allergies, clinician documentation detailing the nature and severity of the reaction is often lacking. Of those patients reporting an allergy, only $4 \%$ of documented allergies had a specific type of reaction provided in the medical chart [1].

It is estimated that about $15.6 \%$ of patients report having a beta-lactam allergy, however, estimates are much lower for patients that actually exhibit a severe allergic reaction preventing their use [2]. Skin testing, in vivo radioallergosorben testing (RAST), and provocation tests can be done to diagnose a true drug hypersensitivity $[2,3]$. In a study evaluating patients with a history of cephalosporin allergy, only $40.1 \%$ of allergies were confirmed by these methods [3]. Although oral provocation testing is considered the gold standard in diagnosis, it is infrequently used due to the need for close observation and risk of severe uncontrollable reactions [4]. In children with a history of a penicillin or cephalosporin allergy, only $58.3 \%$ of allergies were confirmed with positive skin or challenge testing [5]. In practice, many clinicians avoid beta-lactam antibiotics if there is a reported allergy even without the documented reaction [1]. These reported allergies may have a significant impact on treatment when antibiotics considered second line agents or those associated with increased toxicity are used instead of preferred agents.

Because of the fear of cross-reactivity between related classes of antibiotics, patients with allergies to beta-lactams are prescribed alternative antibiotics such as vancomycin, aminoglycosides, and fluoroquinolones with increased frequency $[1,2]$. A study of patients with suggested beta-lactam allergies demonstrated that $30.3 \%$ of these patients received antibiotic therapies different than the standard of care [2]. These patients were treated with antibiotics for a variety of indications including pulmonary, urinary, skin and soft tissue, blood, intraabdominal, and bone infections as well as prophylaxis. Use of alternative antibiotics can lead to additional concerns of increased 
health care cost, unwanted adverse reactions, and bacterial resistance $[2,6]$. Toxicities of particular concern are those associated with vancomycin or aminoglycosides and fluoroquinolones including nephrotoxicity and Clostridium difficile-associated diarrhea, respectively. Not only is there concern for nephrotoxicity with vancomycin, but the use of vancomycin for the trea-tment of invasive methicillinsusceptible Staphylococcus aureus invasive MSSA infections is inferior when compared to traditional first line agents such as anti-staphylococcal penicillins and first generation cephalosporins. Studies have shown that treatment with vancomycin for MSSA infections may result in higher rates of treatment failure and relapse $[7,8]$.

Of particular concern for healthcare providers is the problem of cross reactivity between penicillins and other beta-lactams, including cephalosporins and carbapenems. While these antibiotics share the same lactam ring structure, which was once thought to be the cause of shared hypersensitivity reactions, research has shown that it is more likely the side chains that drive the shared sensitivity. Therefore the actual incidence of cross-reactivity is likely much lower than often feared, with reports of less than $2 \%$ cross-reactivity if the side chains are different $[9,10]$.

Desensitization is an effective option for a patient that may require treatment with a specific or related agent to which they are allergic. Additionally, desensitization may be used in serious infections such as neurosyphilis, when there is a known inferior activity of the second line agent [11]. Desensitization should be considered whenever a patient has an allergic reaction to the first line agent and alternative agents are less effective, such as invasive Staphylococcus aureus infections, where a second line agent such as vancomycin in a methicillin-sensitive strain, may be inferior to an anti-staphylococcal penicillin or first generation cephalosporin. While there are some risks associated with desensitization, when performed in a controlled setting, it is found to be a safe option for patients with a life-threatening infection [12].

We report a case of successful cefazolin desensitization in an aminopenicillin and cephalosporin-allergic patient with a documented invasive MSSA infection who was clinically unresponsive to vancomycin therapy. We describe the potential advantages for the use of desensitization protocols in commonly encountered Staphylococcal infections.

\section{Case}

A 64 year old Caucasian man presented to the hospital with lower extremity pain and petechiae. Past medical history was significant for hypertension, coronary artery disease status post right coronary artery stent 2 years prior, congestive heart failure, stage IV chronic kidney disease, and thrombocytopenia. Two weeks prior to admission, he was prescribed amoxicillin for what he described as a "cold." One week prior to admission, his platelets were $19 \times 10^{3} /$ $\mathrm{uL}$ and he was subsequently started on prednisone $60 \mathrm{mg}$ orally. Pertinent laboratory values on admission included a white blood cell count $18 \times 10^{3} / \mathrm{uL}\left(3.6-9.3 \times 10^{3} / \mathrm{uL}\right)$, platelets $41 \times 10^{3} / \mathrm{uL}\left(150-450 \times 10^{3} / \mathrm{uL}\right)$, BUN $46 \mathrm{mg} / \mathrm{dl}(6-20 \mathrm{mg} / \mathrm{dl})$, serum creatinine $1.7 \mathrm{mg} / \mathrm{dl}(0.9-1.3 \mathrm{mg} / \mathrm{dl})$, and glucose $319 \mathrm{mg} / \mathrm{dl}$ (70-99 mg/dl). All other laboratory values were within normal range. Physical exam was significant for left lower extremity petechiae that were tender to palpitation. Allergies documented in the medical chart at admission include amoxicillin and cefotetan. The admitting physician reported in the history and physical that both drugs caused itching and that amoxicillin could have been the cause of his thrombocytopenia as well.

On hospital day 2, the patient was unable to void, and a foley catheter was inserted. The patient was febrile (Tmax $=101.2^{\circ} \mathrm{F}$ ), and 2 of 2 blood cultures and dialysis catheter tip cultures revealed gram positive cocci, which were later identified as MSSA (resistant only to clindamycin and penicillin G). Vancomycin 1 gram every 12 hours (14 mg/ $\mathrm{kg}$ ) and meropenem $500 \mathrm{mg}$ every 8 hours were empirically started on day 2. The meropenem was discontinued on day 3 to streamline therapy. The patient's initial vancomycin trough concentration was $21 \mathrm{mg} / \mathrm{L}$, however the patient's serum creatinine increased to $2 \mathrm{mg} / \mathrm{dl}$ on day 8 of hospitalization, and at that time the vancomycin trough increased to $41 \mathrm{mg} / \mathrm{L}$. Vancomycin doses were held for the next 6 days. The patient's vancomycin trough decreased to $10 \mathrm{mg} / \mathrm{L}$ and his serum creatinine improved to $1.7 \mathrm{mg} /$ $\mathrm{dl}$, and the vancomycin was restarted at $750 \mathrm{mg}$ daily. The patient's signs and symptoms improved and was discharged on hospital day 16 after completing 14 days of vancomycin therapy for his MSSA bacteremia.

Less than 72 hours after discharge, the patient presented again to the hospital with increasing lower extremity edema and temperature of $101^{\circ} \mathrm{F}$. Pertinent laboratory values on admission included a white blood cell count $12 \times 10^{3} / \mathrm{uL}$ $\left(3.6-9.3 \times 10^{3} / \mathrm{uL}\right)$, hemoglobin $8.1 \mathrm{~g} / \mathrm{dl}(12-17.8 \mathrm{~g} / \mathrm{dl})$, platelets $65 \times 10^{3} / \mathrm{uL}$ (150-450 x 103/uL), BUN $53 \mathrm{mg} / \mathrm{dl}(6-20 \mathrm{mg} / \mathrm{dl})$, serum creatinine $2 \mathrm{mg} / \mathrm{dl}(0.9-1.3 \mathrm{mg} / \mathrm{dl})$, and glucose 218 $\mathrm{mg} / \mathrm{dl}(70-99 \mathrm{mg} / \mathrm{dl})$. All other laboratory values were within normal range. Physical exam findings were significant for bilateral lower extremity pitting edema, erythema from mid calf to ankle of left lower extremity, and petechial rash on his upper extremities. Two of 2 blood cultures on day 1 of the second hospitalization grew gram positive cocci, later identified as MSSA (resistant only to clindamycin, penicillin $\mathrm{G}$, and erythromycin). Infectious diseases consult on day 2 of hospitalization recommended continuing vancomycin due to the documented reaction of severe itching with penicillin. Because of the apparent recurrence of bacteremia following 2 weeks of vancomycin therapy, there was concern for an unknown source such as an abscess, osteomyelitis, or endocarditis and further work-up was performed. A transesophageal echocardiogram was negative for vegetations and a bone scan showed no evidence of osteomyelitis. Surgery was consulted and their impression included soft 
Cash et al. Journal of Pharmaceutical Technology \& Drug Research 2013, http://www.hoajonline.com/journals/pdf/2050-120X-2-15.pdf

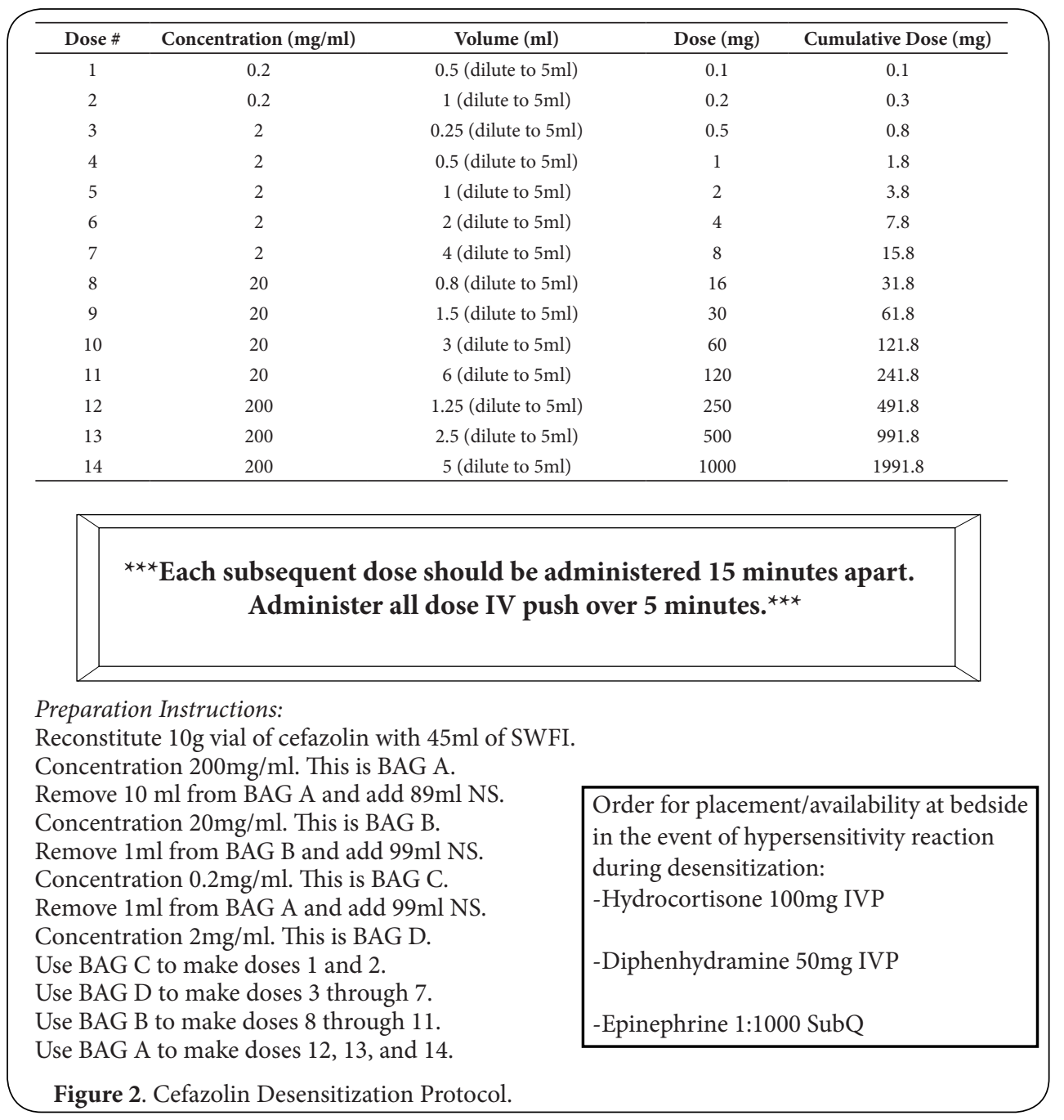

tissue abscess in the lower leg based on physical exam findings of area of fluctuance in the lateral aspect of the mid calf with erythema and mild induration. On day 4 of hospitalization and day 3 of vancomycin therapy, the patient continued to have persistent bacteremia with repeat blood cultures positive for MSSA (resistant only to clindamycin, penicillin $G$, and erythromycin). On day 5 , surgical debridement of the abscesses was performed. Wound cultures confirmed the presence of MSSA. Also on day 5 of hospitalization, the infectious diseases team recommended cefazolin desensitization followed by a 2-week course of cefazolin because of persistent bacteremia (Figure 2). Desensitization was recommended due to the patient's history of amoxicillin and cefotetan allergies. The patient tolerated the desensitization protocol and was continued on cefazolin 1 gram every 8 hours for 14 days. The patient improved significantly with surgical debridement and cefazolin therapy with normalization of white count and fever curve. Repeat blood cultures on day 18 of hospitalization revealed no growth. The patient was discharged after 19 days of hospitalization to complete a 10-day course of levofloxacin 500 mg daily. (Figure 1) represents a timeline of the patient's hospitalizations.

\section{Discussion}

Our case demonstrates the usefulness of desensitization in a patient with a history of presumed IgE mediated reaction to amoxicillin and a documented staphylococcal infection, sensitive to cefazolin. Desensitization can be useful in providing preferred treatment options, if it has been determined that a patient has a true hypersensitivity to an essential drug, and the reaction is IgE-mediated. Examples of IgE-mediated reactions include anaphylaxis, urticaria, and bronchospasm [6]. Our patient had a reported allergy to both amoxicillin and cefotetan and was successfully desensitized to cefazolin.

By administering an antibiotic agent in increasing concentrations slowly over time, the patient can develop a clinical tolerance and become desensitized to the agent, without precipitating further IgE-mediated reactions [13]. Desensitization can be done over a few hours via rapid desensitization, or over a few days via slow desensitization 


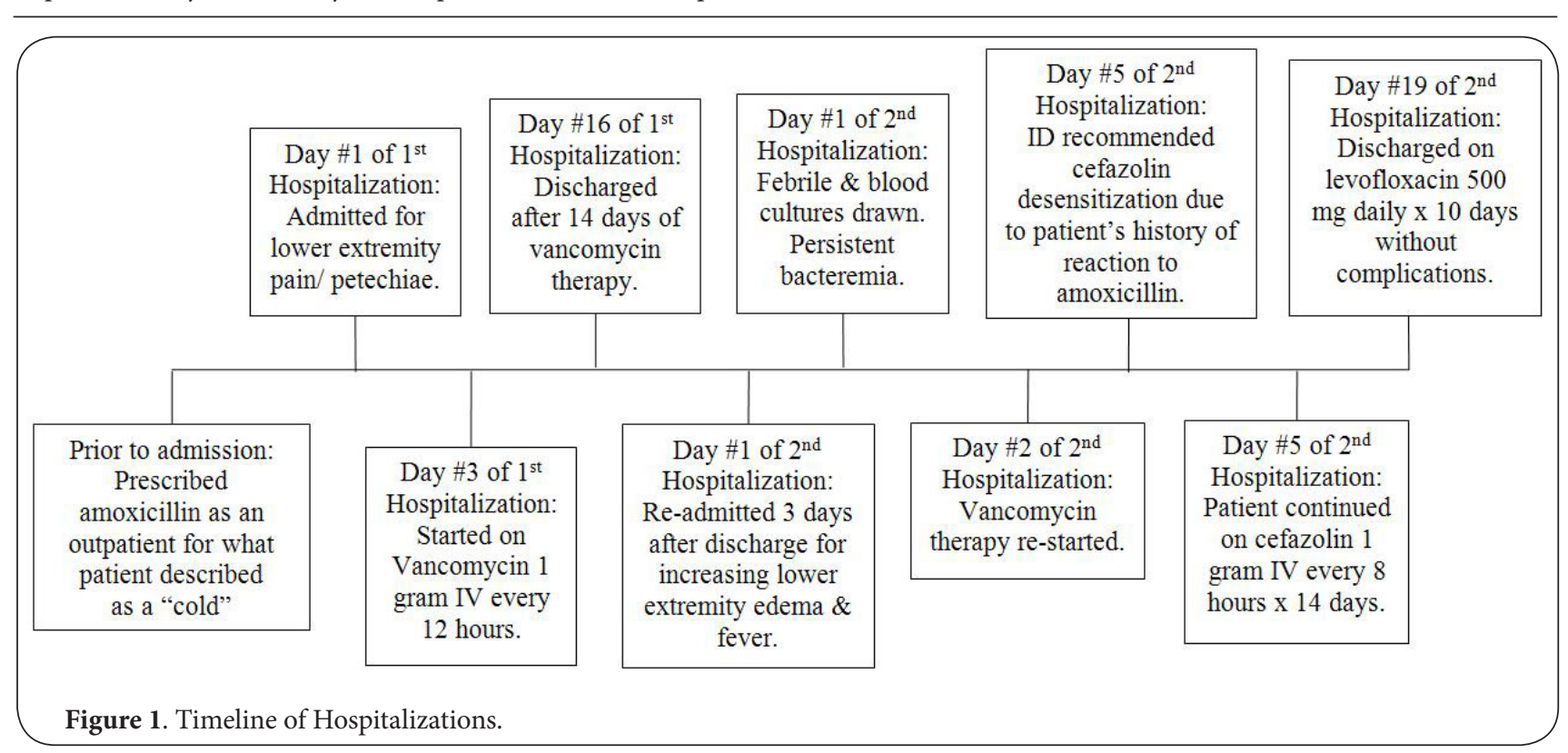

$[13,14]$. See (Figure 2) for the cefazolin desensitization protocol used in this patient case.

In rapid protocols, the strength of the drug begins with $1 / 10000$ to $1 / 100$ of the target dose (usually in the mcg range), and the dose is increased every 10-30 minutes. $A$ period of 100-300 minutes is necessary to complete the entire protocol and get the patient to therapeutic dosing. The general principle is the same for both oral and IV procedures, but oral is considered safer, causing fewer reactions $[13,14]$.

When performed correctly and under supervision, desensitization can be a safe and effective way to administer optimal antimicrobial treatment in patients with confirmed infections and known allergies. Desensitization has been well studied in cystic fibrosis patients given the multiple and repeat exposure to antimicrobials, coupled with the high incidence of reported antimicrobial allergies. In these patients, beta-lactam allergies can be present in as many as $36-60 \%$ of the population [15]. One study performed 52 desensitizations on 15 patients with cystic fibrosis with most desensitizations performed with piperacillin or ceftazidime [16]. The majority of the desensitizations (96.4\%) were successful with no serious adverse reactions preventing the completion of desensitization. Two patients experienced serious adverse reactions. One patient had nausea, diarrhea, flushing, and an erythematous rash and the other patient developed acute respiratory failure requiring intubation. The first patient was successfully re-desensitized. In another study by Turvey and colleagues, $75 \%$ of desensitizations were successful and of those $86 \%$ reported no reactions and $14 \%$ reported mild cutaneous reactions [17]. Evidence of systemic allergic reactions occurred in 19\% of the remaining patients. None of these patients required intubation and there were no fatalities reported. The remaining patients discontinued desensitization for reasons other than an allergic reaction.

While desensitization has proven efficacy, there are some risks associated with desensitization. For example, mild allergic reactions occur in $30 \%$ of patients [18]. Mild reactions can include flushing, pruritus, and rashes. These can be managed by slowing the infusion or by administering antihistamines and/or corticosteroids. More serious reactions include severe asthma, hypotension, laryngeal edema, and anaphylaxis. Presentation of these reactions are much rarer, but do occasionally occur. Among patients with severe reactions, the protocol may still be completed. If a patient shows signs of these reactions, treatment must be stopped temporarily [18]. Once the patient is stable, desensitization can be resumed at one-third the previous dose $[\mathbf{1 4}, \mathbf{1 8}]$. Because this is a high risk procedure that poses a risk for anaphylactic reactions, the patient should be under continuous cardiac monitoring with frequent nursing assessments of respiratory status and signs of reaction including itching. Certain medications and equipment should always be on hand in case the patient does experience an anaphylactic reaction including epinephrine, vasopressive drugs, full resuscitative measures, and suctioning [18]. Per protocol in our institution, diphenhydramine, hydrocortisone, and epinephrine were kept at bedside. Our patient did have to repeat one dose of cefazolin during the protocol and received one dose of diphenhydramine because of hoarseness and scratching in his throat. Otherwise our patient tolerated the protocol well. After desensitization has been completed, and the optimal dose has been reached, the patient can continue treatment. If treatment is stopped or interrupted for more than 12 hours, the patient may have to go through the desensitization process again $[18,19]$. There are times in which desensitization should be avoided in patients. For example, those who have encountered life-threatening non- 
IgE-mediated reactions such as Stevens-Johnson or toxic epidermal necrolysis should not attempt desensitization [20].

Cross-reactivity between penicillins and cephalosporins with identical side chains can be as high as $30 \%$, however if the side chains are different, cross-reactivity drops to less than $2 \%[9,19]$. Cefazolin, cefuroxime, and ceftriaxone are examples of cephalosporins that have different side chains from amoxicillin and therefore are expected to have a low incidence of cross-reactivity [21]. The incidence of crossreactivity of penicillins and carbapenems is reported to be 0.9 to $11 \%$, with one isolated study finding the incidence to be as high as $47.4 \%[6,22,23]$. Our patient tolerated a 2 -day course of meropenem. A review of 11 studies found that about $4.4 \%$ of patients with a positive penicillin skin test would be at an increased risk of a reaction with a cephalosporin. Also, patients with a history of a penicillin allergy but a negative skin test had a much lower risk of reaction with a cephalosporin [24].

Desensitization may be particularly beneficial in staphylococcal infections due to the significant morbidity and mortality associated with both MSSA and methicillinresistant Staphylococcus aureus (MRSA). MSSA remains a common cause of invasive infections and is even linked to more deep-seated infections compared to MRSA [25]. An increased incidence of complications from staphylococcal bacteremia has been associated with several risk factors, such as community-acquired infection, suspected acute systemic infection, persistent fever, and positive blood culture follow-up [26].

Vancomycin is often considered to be first line treatment against MRSA. Unfortunately, this also leads to increased use for MSSA infections, as it is often initiated empirically before the results of susceptibility testing are known. Increased use of this drug in the treatment of MSSA has been linked with higher morbidity and mortality [27]. In addition to these problems, the use of vancomycin in place of cephalosporins has often been linked to increased relapse and hospital readmission, as well as an increased incidence of endocarditis [8]. The mortality rate was found to be $23 \%$ for patients started on empiric vancomycin and then switched to an antistaphylococcal penicillin for MSSA compared to a mortality rate of $12 \%$ of patients that were started on penicillin initially [28].

In contrast to vancomycin, beta-lactam antibiotics, particularly cephalosporins, demonstrate greater efficacy against MSSA. In a study by Stryjewsi and colleagues, patients treated with cefazolin showed more favorable outcomes versus vancomycin, although patients had more pervasive infections at time of presentation. Patients taking cefazolin had a $91.3 \%$ cure rate, compared to $83.1 \%$ in patients taking vancomycin [7]. The patients treated with vancomycin also experienced higher rates of treatment failure than those taking cefazolin, at $31.2 \%$ vs. $13 \%$, respectively [7]. Vancomycin was also linked in another study to early relapse as well as increased duration of infection in patients with indwelling intravascular devices [29]. In a retrospective, matched case control study by Kim and colleagues, the authors examined two treatment groups for MSSA bacteremia, a vancomycin group and betalactam group. This study found that bacteremia-related mortality and 14-day mortality were statistically higher in the vancomycin group compared to the beta-lactam group [30]. Patients with MSSA infections treated with vancomycin were at higher risk of bacteremia-related death than those receiving beta-lactams [30]. This study, as well as others, demonstrate the superiority of beta-lactams in the treatment of MSSA and the necessity of their use to fight infection.

Our patient did benefit from the initiation of a betalactam, cefazolin, for an invasive MSSA infection. By desensitizing our patient we were able to utilize a preferred therapy.

\section{Conclusion}

For patients that have a true allergy to penicillin, desensitization should be a consideration in treatment, especially in invasive infections, where mortality rates remain high and second-line agents are inferior. The use of appropriate antibiotics initially is important for the welfare of our patients and when performed appropriately desensitization can be a safe alternative with minimal risk for serious adverse reactions.

\section{Competing interests}

P. Brandon Bookstaver has received research money from Merck and Cubist.

Acknowledgement

Amanda Zomp, PharmD, PGY1 pharmacy resident at Palmetto Health Richland at the time of study.

\section{Publication history}

Received: 01-Jan-2013 Revised: 01-Feb-2013

Re-Revised: 05-Mar-2013 Accepted: 21-Mar-2013

Published: 15-Apr-2013

\section{References}

1. Lee CE, Zembower TR, Fotis MA, Postelnick MJ, Greenberger PA, Peterson LR and Noskin GA: The incidence of antimicrobial allergies in hospitalized patients: implications regarding prescribing patterns and emerging bacterial resistance. Arch Intern Med 2000, 160:281922. | Article | PubMed

2. Lutomski DM, Lafollette JA, Biaglow MA and Haglund LA: Antibiotic allergies in the medical record: effect on drug selection and assessment of validity. Pharmacotherapy 2008, 28:1348-53. | Article I PubMed

3. Antunez C, Blanca-Lopez N, Torres MJ, Mayorga C, Perez-Inestrosa $\mathrm{E}$, Montanez MI, Fernandez T and Blanca M: Immediate allergic reactions to cephalosporins: evaluation of cross-reactivity with a panel of penicillins and cephalosporins. J Allergy Clin Immunol 2006, 117:404-10. | Article | PubMed

4. Bousquet PJ, Gaeta F, Bousquet-Rouanet L, Lefrant JY, Demoly P and Romano A: Provocation tests in diagnosing drug hypersensitivity. Curr Pharm Des 2008, 14:2792-802. I Article I PubMed

5. Atanaskovic-Markovic M, Velickovic TC, Gavrovic-Jankulovic M, Vuckovic $\mathrm{O}$ and Nestorovic B: Immediate allergic reactions to cephalosporins and penicillins and their cross-reactivity in children. 
Pediatr Allergy Immunol 2005, 16:341-7. | Article | PubMed

6. Frumin J and Gallagher JC: Allergic cross-sensitivity between penicillin, carbapenem, and monobactam antibiotics: what are the chances? Ann Pharmacother 2009, 43:304-15. | Article | PubMed

7. Stryjewski ME, Szczech LA, Benjamin DK, Jr., Inrig JK, Kanafani ZA, Engemann JJ, Chu VH, Joyce MJ, Reller LB, Corey GR and Fowler VG, Jr.: Use of vancomycin or first-generation cephalosporins for the treatment of hemodialysis-dependent patients with methicillinsusceptible Staphylococcus aureus bacteremia. Clin Infect Dis 2007, 44:190-6. | Article | PubMed

8. Chang FY, MacDonald BB, Peacock JE, Jr., Musher DM, Triplett $P$, Mylotte JM, O'Donnell A, Wagener MM and Yu VL: A prospective multicenter study of Staphylococcus aureus bacteremia: incidence of endocarditis, risk factors for mortality, and clinical impact of methicillin resistance. Medicine (Baltimore) 2003, 82:322-32. Article | PubMed

9. Moreno E, Macias E, Davila I, Laffond E, Ruiz A and Lorente F: Hypersensitivity reactions to cephalosporins. Expert Opin Drug Saf 2008, 7:295-304. | Article | PubMed

10. Robinson JL, Hameed T and Carr S: Practical aspects of choosing an antibiotic for patients with a reported allergy to an antibiotic. Clin Infect Dis 2002, 35:26-31. | Article | PubMed

11. Brown DL, Frank JE. Diagnosis and management of syphillis. Am Fam Physician. 2003, 68:283-90,297. | Article

12. Cernadas JR, Brockow K, Romano A, Aberer W, Torres MJ, Bircher A, Campi P, Sanz ML, Castells M, Demoly P and Pichler WJ: General considerations on rapid desensitization for drug hypersensitivity - a consensus statement. Allergy 2010, 65:1357-66. | Article | PubMed

13. Gruchalla RS, Pirmohamed M: Clinical practice. Antibiotic allergy. $N$ Engl J Med. 2006; 354:601-9. | PubMed | Article

14. Castells M: Rapid desensitization for hypersensitivity reactions to medications. Immunol Allergy Clin North Am 2009, 29:585-606. | Article | PubMed

15. Burrows JA, Nissen LM, Kirkpatrick CM and Bell SC: Beta-lactam allergy in adults with cystic fibrosis. J Cyst Fibros 2007, 6:297-303. I Article | PubMed

16. Legere $\mathrm{HJ}, 3 \mathrm{rd}$, Palis RI, Rodriguez Bouza T, Uluer AZ and Castells MC: A safe protocol for rapid desensitization in patients with cystic fibrosis and antibiotic hypersensitivity. J Cyst Fibros 2009, 8:418-24. | Article | PubMed Abstract | PubMed Full Text

17. Turvey SE, Cronin B, Arnold AD and Dioun AF: Antibiotic desensitization for the allergic patient: 5 years of experience and practice. Ann Allergy Asthma Immunol 2004, 92:426-32. | Article | PubMed

18. Candela L: Caring for a patient with Listeria endocarditis: use of antibiotic desensitization. Crit Care Nurse 2002, 22:38-43. | Article | PubMed

19. Arroliga $\mathrm{ME}$ and Pien L: Penicillin allergy: consider trying penicillin again. Cleve Clin J Med 2003, 70:313-4, 317-8, 320-1 passim. | Article I PubMed

20. de Groot $\mathrm{H}$ and Mulder WM: Clinical practice: drug desensitization in children. Eur J Pediatr 2010, 169:1305-9. | Article | PubMed Abstract I PubMed Full Text

21. Novalbos A, Sastre J, Cuesta J, De Las Heras M, Lluch-Bernal $\mathrm{M}$, Bombin $\mathrm{C}$ and Quirce $\mathrm{S}$ : Lack of allergic cross-reactivity to cephalosporins among patients allergic to penicillins. Clin Exp Allergy 2001, 31:438-43. | Article | PubMed

22. Sodhi M, Axtell SS, Callahan J and Shekar R: Is it safe to use carbapenems in patients with a history of allergy to penicillin? J Antimicrob Chemother 2004, 54:1155-7. | Article | PubMed

23. Prescott WA, Jr., DePestel DD, Ellis JJ and Regal RE: Incidence of carbapenem-associated allergic-type reactions among patients with versus patients without a reported penicillin allergy. Clin Infect Dis 2004, 38:1102-7. | Article | PubMed

24. Kelkar PS and Li JT: Cephalosporin allergy. N Engl J Med 2001, 345:804-9. | Article | PubMed |

25. Sattler CA, Mason EO, Jr. and Kaplan SL: Prospective comparison of risk factors and demographic and clinical characteristics of community-acquired, methicillin-resistant versus methicillinsusceptible Staphylococcus aureus infection in children. Pediatr Infect Dis J 2002, 21:910-7. | Article | PubMed

26. Corey GR: Staphylococcus aureus bloodstream infections: definitions and treatment. Clin Infect Dis 2009, 48 Suppl 4:S254-9. | Article | PubMed

27. Mongkolrattanothai K, Aldag JC, Mankin P and Gray BM: Epidemiology of community-onset Staphylococcus aureus infections in pediatric patients: an experience at a Children's Hospital in central Illinois. BMC Infect Dis 2009, 9:112. | Article | PubMed Abstract | PubMed Full Text

28. Brown J and Paladino JA: Impact of rapid methicillin-resistant Staphylococcus aureus polymerase chain reaction testing on mortality and cost effectiveness in hospitalized patients with bacteraemia: a decision model. Pharmacoeconomics 2010, 28:56775. | Article | PubMed

29. Hartstein Al, Mulligan ME, Morthland VH and Kwok RY: Recurrent Staphylococcus aureus bacteremia. J Clin Microbiol 1992, 30:670-4. | Pdf | PubMed Abstract | PubMed Full Text

30. Kim SH, Kim KH, Kim HB, Kim NJ, Kim EC, Oh MD and Choe KW: Outcome of vancomycin treatment in patients with methicillinsusceptible Staphylococcus aureus bacteremia. Antimicrob Agents Chemother 2008, 52:192-7. | Article | PubMed Abstract | PubMed Full Text

\section{Citation:}

Cash J H, Caulder C R, Quidley A M, Frith M C, Davenport A and Bookstaver P B: Desensitization as a tool for beta-lactam antibiotic use in methicillin sensitive Staphylococcus aureus infections. journal of Pharmaceutical Technology and Drug Research 2013, 2:15. http://dx.doi.org/10.7243/2050-120X-2-15 